\title{
Uso de registros de assistência farmacêutica do Sistema de Informações Ambulatorial para avaliação longitudinal de utilização e adesão a medicamentos
}

\author{
Use of pharmaceutical care programs \\ records of the Outpatient Information System for the \\ assessment of patient's medication use and adherence
}

Claudia Soares ${ }^{1}$, Gulnar Azevedo Silva²

\begin{abstract}
Resumo
Objetivo: Descrever o processo de criação de uma coorte de pacientes a partir dos registros de reabastecimento de receitas de um programa de assistência farmacêutica. Métodos: Coorte retrospectiva de pacientes admitidos num programa de assistência em doença de Alzheimer entre 2002 e 2005 no estado do Rio de Janeiro. Os registros de reabastecimento de receitas saíram dos dados de Autorização de Procedimentos Ambulatoriais de Alta Complexidade do Sistema de Informações Ambulatoriais do Sistema Único de Saúde. Foi utilizado o relacionamento probabilístico de dados para identificação das múltiplas entradas de cada indivíduo e o programa estatístico Statistical Package for the Social Sciences (SPSS) para construção da coorte e das medidas. Resultados: Foi obtida a base cuja unidade de análise é o indivíduo, contendo os dados demográficos, unidade de dispensação, as múltiplas entradas de abastecimento com suas respectivas datas de apresentação, código primário e secundário do CID, o procedimento realizado (nesse caso medicamento e dose por unidade de apresentação) e número de unidades dispensadas. Conclusões: A base criada com dados secundários permite a avaliação do perfil de utilização e exposição a medicamentos e amplia as possibilidades de pesquisa na área farmacoepidemiologia e avaliação de programas.

Palavras-chave: adesão à medicação; farmacoepidemiologia; avaliação de programas e projetos de saúde; sistemas de informação em saúde; registro médico coordenado.

Abstract

Objective: To describe the process of creating a cohort from refill data from a pharmaceutical assistance program and evaluate the feasibility of using such data in the construction of measures that assess patients' use and adherence to this program. Methods: Retrospective cohort of patients admitted to a pharmaceutical care program in Alzheimer's disease between 2002 and 2005 in the state of Rio de Janeiro. The refill data records were taken from the Authorization of High Complexity Procedures of the Outpatient Information System of the Unified Health System. The probabilistic record linkage methodology was used to identify the multiple entries for each individual and the statistics software Statistical Package for the Social Sciences (SPSS) to build the cohort. Results: After completion of all processing steps, a database was obtained where the unit of analysis was the individual, containing demographics, dispensing unit, the multiple supply with their respective dates of submission, primary and secondary ICD codes, the performed procedures (in this case medications and dose per unit) and the number of units dispensed. Conclusions: The formed measures allow the assessment of medicines utilization and exposure using secondary data as unique information source, plus open new possibilities in the pharmacoepidemiology and healthcare program evaluation field.
\end{abstract}

Keywords: medication adherence; pharmacoepidemiology; program evaluation; health information systems; medical record linkage.

Trabalho realizado no Instituto de Estudos em Saúde Coletiva da Universidade Federal do Rio de Janeiro (IESC/UFRJ) - Rio de Janeiro (RJ), Brasil.

'Doutora em Saúde Pública pelo Instituto de Medicina Social da Universidade do Estado do Rio de Janeiro (UERJ) - Rio de Janeiro (RJ), Brasil.

${ }^{2}$ Professora Adjunta do Departamento de Epidemiologia do Instituto de Medicina Social da UERJ - Rio de Janeiro (RJ), Brasil.

Endereço para correspondência: Cláudia Soares - Estrada dos Bandeirantes, 8464 - CEP: 22783-110 - Rio de Janeiro (RJ), Brasil -

E-mail: claudia.sr@globo.com

Fonte de financiamento: nenhuma.

Conflito de interesses: nada a declarar. 


\section{INTRODUÇÃO}

Segundo a Organização Mundial de Saúde (OMS), adesão ao tratamento é:

a medida com que o comportamento de uma pessoa tomar a sua medicação, seguir a dieta e/ ou mudar seu estilo de vida - corresponde às recomendações de um profissional de saúde ${ }^{1}$.

Portanto, esse fenômeno da adesão ao tratamento está relacionado não só ao uso de medicamentos, como a todos os aspectos do cuidado, desde seguir recomendações de mudanças de estilo de vida até comparecer às consultas ou ter acesso ao tratamento. Este fenômeno resulta da inter-relação de um conjunto de fatores individuais, do sistema de saúde e do próprio tratamento em $\mathrm{si}^{1} \mathrm{e}$ apresenta uma relação direta com os desfechos e custos na saúde $\mathrm{e}^{2,3}$. Portanto, o monitoramento das taxas de adesão se apresenta como um elemento chave na avaliação de qualidade e desempenho de um programa público de assistência.

Apesar da sua reconhecida importância na saúde pública ${ }^{4}$ e altos gastos relacionados ${ }^{5,6}$, pouco se tem estudado a respeito da adesão aos medicamentos cobertos nos programas de assistência farmacêutica de alto custo ${ }^{7}$. Uma exceção é o trabalho realizado no contexto de tratamento da AIDS pela Secretária de Vigilância em Saúde, orientado a uma melhor compreensão dos fatores associados e ao planejamento de ações para melhora da adesão de antirretrovirais ${ }^{8,9}$.

Pela complexidade que engloba, não há uma técnica padrão ouro que possa medir o comportamento de adesão ao tratamento e várias técnicas têm sido discutidas na literatu$\mathrm{ra}^{10-13}$. Entre elas, os estudos de reabastecimento de receitas em bases de dados administrativas têm sido frequentemente utilizados fora do Brasil, principalmente, pela possibilidade de estudar grandes populações, num intervalo curto de tempo. Esses estudos se apoiam no fato de cada vez que um indivíduo procura o serviço de saúde para receber a medicação prescrita, ou seja, reabastecer sua receita, ele gera um registro numa base de dados e, através da repetição desses registros, pode-se acompanhar a experiência desses indivíduos com o tratamento de maneira longitudinal. No entanto, alguns fatores podem influenciar a capacidade de uma base de dados em gerar medidas de adesão de maneira adequada: qualidade dos dados, como o número de campos ausentes ou mal preenchidos e o tipo de dado presente na base, como medicamento prescrito, dose e frequência de reabastecimento. Outra questão é a forma que a base de dados está estruturada. Em uma base relacional, os dados necessários para a pesquisa podem estar disponíveis em diferentes arquivos de dados, o que aumenta a necessidade de conhecimento em programação. Além disso, dependendo do objetivo da pesquisa, a forma de entrada e codificação do dado podem também afetar a validade da informação.

No Brasil, essa metodologia de reabastecimento de receitas já foi aplicada em pacientes em uso de antirretroviral com os dados das bases de dados do Sistema de Controle Logístico de Medicamentos (SICLOM) ${ }^{14}$, apresentando resultados importantes para a vigilância dos pacientes portadores de AIDS. Os resultados desse estudo apontaram para altas proporções de retiradas irregulares dos medicamentos $(57,9 \%)$ e de abandono do tratamento $(30,3 \%)$.

Outra base de dados com ampla cobertura populacional que possui dados de uso de medicações e com potencial para a avaliação de indicadores de utilização de medicamentos ou programas é o subsistema Autorização de Procedimentos de Alta Complexidade (APAC) do Sistema de Informação Ambulatorial do Sistema Único de Saúde (SIA-SUS). A inclusão dos Medicamentos de Dispensação em Caráter Excepcional na tabela de valores dos procedimentos do Sistema de Informações Ambulatoriais do Sistema Único de Saúde ocorreu inicialmente em outubro de 1993 e, desde então, várias portarias incluindo ou excluindo medicamentos já ocorreram.

O principal objetivo dessa base é a coleta de informações que permitam aos prestadores de saúde informar sua produção e, consequentemente, a efetivação do pagamento pelos serviços prestados no SUS. Essa base de dados inclui informação dos procedimentos de alta complexidade que, após a aprovação realizada por médicos avaliadores, são disponibilizados aos usuários através de programas de assistência.

Nos programas que englobam os componentes especializados da assistência farmacêutica, o acesso ao medicamento ocorre através do preenchimento do laudo de solicitação de medicamentos (LME) pelo médico assistente. A autorização do LME é efetivada somente após o deferimento da avaliação, realizada em conformidade com os Protocolos Clínicos publicados pelo Ministério da Saúde ${ }^{15}$. Após isso, todos os dados da LME são digitados no instrumento de Autorização de Procedimentos Ambulatoriais de Alta Complexidade e a dispensação do medicamento ao paciente ocorre mensalmente por um período de três meses (vigência da APAC). Após esse prazo, o processo deve ser renovado. Logo, cada APAC pode

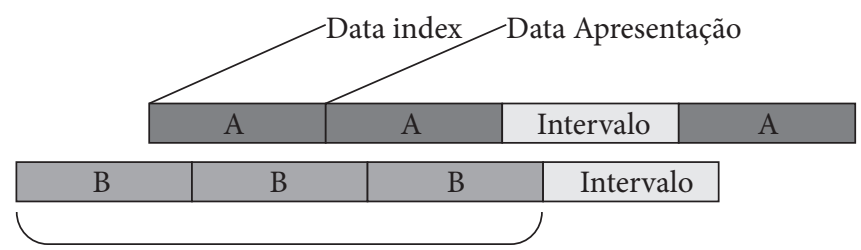

3 abastecimentos $=$ Uma APAC

Figura 1. Esquema representativo do reabastecimento de receitas (A/B) na base de dados da Autorização de Procedimentos Ambulatoriais de Alta Complexidade e respectivos marcos temporais 
representar a entrada de até três registros de dados de abastecimento (Figura 1) e o mesmo indivíduo pode dar entrada em vários procedimentos e, portanto, gerar várias APAC ao longo de um mesmo ano. Isso resulta em uma relação um-para-muitos, de múltiplos registros para o mesmo indivíduo.

Todos esses registros são capturados pelos municípios e estados e enviados em remessas mensais para serem consolidados pelo Departamento de Informática do SUS (DATASUS). O processamento das informações é também realizado em múltiplos arquivos com estruturas diversas que após consolidação e validação dos dados, ficam disponíveis para análise pelo Ministério da Saúde.

Essas características, embora facilitem a avaliação administrativa e financeira, dificultam uma análise da base de dados quando o acompanhamento individual se faz necessário, pois, nesse caso, uma série de procedimentos específicos de integração e transformação de dados é requerida.

O objetivo deste estudo é descrever o processo de criação de uma coorte de pacientes a partir dos registros de reabastecimento de receitas de um programa de assistência farmacêutica e avaliar a viabilidade do uso desses dados na construção de medidas que estimem a adesão dos pacientes a esse programa.

\section{MÉTODOS}

Para a criação da base de dados de estudo, foram utilizados os arquivos identificados de pacientes que recebem medicamentos excepcionais do subsistema APAC do Sistema de Informação Ambulatorial (SIA) do Estado do Rio de Janeiro referentes ao período de 2002 a 2005. Os arquivos foram cedidos pela Coordenadoria Geral de Sistemas de Informação do Ministério da Saúde (Ofício nº 169/2012/DRAC/SAS/MS em 28/05/2012) para uso conforme termo de compromisso e responsabilidade firmado pelos investigadores, sempre visando preservar a confidencialidade dos dados.

Dentre os programas passíveis de serem avaliados, optou-se pelo programa orientado para Demência de Alzheimer implementado no Brasil, através das portarias assinadas em abril de 2002 (n. 255, 702, 703 e 249) pelo Ministério de Saúde. Esse programa garante aos pacientes portadores de Demência de Alzheimer, com diagnóstico confirmado por especialistas, o acesso à atenção ambulatorial multidisciplinar e ao tratamento medicamentoso com anticolinesterásicos como donepezil e rivastigmina. O período 2002 a 2005 corresponde ao início da dispensação dos medicamentos anticolinesterásicos no SUS com a possibilidade de seguimento dos pacientes por até três meses.

Como na estrutura desses arquivos os registros estão armazenados por procedimento e não por indivíduos, uma série de etapas foi necessária para a organização desses arquivos de maneira que cada registro representasse o seguimento longitudinal de cada paciente em relação ao acesso e reabastecimento de medicamentos. Essas etapas estão enumeradas a seguir:

1. Transformação dos arquivos da APAC em uma base de dados;

2. Padronização e limpeza dos arquivos;

3. Retirada dos registros duplicados de APAC;

4. Junção dos arquivos PF (cadastro dos pacientes) ao arquivo TA (ocorrências da APAC);

5. Rotina de duplicidade - identificação dos vários registros de um mesmo paciente através da criação de um número único de identificação do paciente;

6. Organização dos registros por paciente.

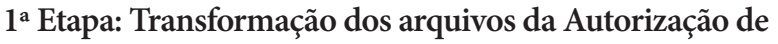 Procedimentos de Alta Complexidade em uma base de dados}

Durante o período selecionado, o subsistema APAC era composto por 11 tipos diferentes de arquivos em formato .dbf, separados por mês e ano de competência:

- AC - Arquivo de APAC dos Pacientes Possui informações sobre o paciente e o procedimento principal autorizado - armazena os dados referentes à cobrança mensal de cada APAC; - PR - Arquivo de Pacientes em Tratamento de Radioterapia - contém registros com informações cadastrais dos pacientes em tratamento de radioterapia;

- PQ - Arquivo de Pacientes em Tratamento de Quimioterapia - contém registros com informações cadastrais dos pacientes em tratamento de quimioterapia;

- CO - Arquivo de Procedimentos Realizados nos Pacientes em Terapia Renal Substitutiva - contém registros com informações cadastrais para cobrança dos procedimentos realizados em Terapia Renal Substitutiva de cada Unidade Prestadora de Atendimentos APAC da rede ambulatorial do SUS;

- UA - Arquivo de Cadastro das Unidades Ambulatoriais contém registros com informações cadastrais de cada Unidade Prestadora de Atendimentos APAC da rede ambulatorial do SUS;

- UO - Arquivo de Cadastro Complementar das Unidades Ambulatoriais - contém registros com informações cadastrais complementares, em tratamentos e equipamentos, de cada Unidade Prestadora de Atendimentos APAC da rede ambulatorial do SUS;

- PC - Arquivo de Pacientes em Terapia Renal Substitutiva contém registros com informações cadastrais dos pacientes em tratamento de terapia renal substitutiva;

- PF - Arquivo de Pacientes que recebem Medicamentos Excepcionais - contém registros com informações cadastrais dos pacientes que recebem medicamentos excepcionais; 
- EX - Arquivo de Resultado de Exames dos Pacientes em Terapia Renal Substitutiva - contém registros com informações cadastrais de resultados de exames dos pacientes que estão em Terapia Renal Substitutiva;

- UD - Arquivo de Cadastro Complementar das Unidades Ambulatoriais de Diálise - contém registros com informações cadastrais complementares de cada Unidade Prestadora de Atendimentos APAC de Diálise da rede ambulatorial do SUS;

- TA - Arquivo de Ocorrências de APAC - contém registros correspondentes a cada autorização de procedimentos ambulatoriais de alta complexidade paga no município no período.

Dos 11 tipos arquivos, apenas 2 tipos armazenavam os dados cadastrais de pacientes e de dispensação de medicamentos necessários para a construção dessa coorte: PF e TA que totalizaram 87 arquivos. Na Tabela 1 estão descritos esses dois tipos de arquivos e as respectivas variáveis de interesse utilizadas nesta análise.

Devido às mudanças que ocorreram na denominação e formatação das variáveis ao longo dos anos, esses arquivos mensais PF precisaram ser padronizados. Nesse processo, cada arquivo foi adaptado de forma a ter o mesmo número e ordem de variáveis (colunas) e cada uma dessas variáveis ser identificada da mesma maneira com o mesmo formato e disposição de dados. Dessa forma, esses arquivos puderam ser integrados através do programa APPENDA do TABWIN, disponível na página eletrônica do DATASUS para uso gratuito ${ }^{16}$. O mesmo processo foi realizado com os arquivos TA, o que resultou em dois arquivos únicos (PF e TA) com todos os dados dos registros APAC do período selecionado para o estudo. Após esse processo, foram selecionados os registros referentes aos procedimentos donepezil e rivastigmina (3631101 a 3631108) que são relacionados ao programa de assistência farmacêutica para doença de Alzheimer.

\section{2a Etapa: Limpeza e Padronização dos arquivos}

Para essa etapa foi necessária, primeiro, a descrição por frequência de todas as variáveis e a identificação de algumas das inconsistências comuns a essas bases de dados, como: campos
CPF com todos os números repetidos (ex.: 0000000000, 99999999999); campos nome da mãe com o valor 'NULO' ou 'Desconhecida' etc.. Foi realizado, em seguida, um processo de limpeza, através do qual inconsistências foram retiradas das bases e esses campos adquiriram, então, um valor nulo e foram considerados como dados ausentes (missing).

Posteriormente foi empregado o programa RecLink $3^{17}$, também disponível eletronicamente para uso gratuito, para padronização dessas bases. Uma descrição mais detalhada do processo de padronização pode ser encontrada no artigo de Queiroz et al. ${ }^{18}$.

\section{$3^{\text {a }}$ Etapa: Retirada dos registros duplicados de Autorização de Procedimentos de Alta Complexidade}

Um problema que pode ocorrer em estudos de bases de dados é a presença de repetições de um mesmo registro, em que dois ou mais registros apresentam todos os campos iguais e correspondem exatamente ao mesmo reabastecimento. Para identificação e exclusão dessas repetições, foi utilizado o comando identify duplicate cases do programa Statistical Package for the Social Sciences (SPSS) 17.0, com as variáveis-chave: número de APAC, data de processamento e número de procedimento principal. A escolha do pacote estatístico SPSS 17.0 deu-se devido à maior familiaridade dos autores com as rotinas de programação do mesmo e adaptações nessas rotinas devem ocorrer em caso de em aplicações futuras dessas técnicas com outros pacotes estatísticos.

\section{4a Etapa: Junção dos arquivos de Pacientes que recebem} Medicamentos Excepcionais ao arquivo de Ocorrências de Autorização de Procedimentos de Alta Complexidade

Primeiro, todos os registros que pertenciam ao mesmo número de APAC e mesmo número de procedimento foram unificados criando uma única linha para cada combinação única. Essa etapa se tornou necessária para que, na posterior unificação dos dados pelo número de APAC, não fossem perdidas as informações do uso associado das medicações (donepezil e rivastigmina) ou da prescrição do mesmo medicamento em doses diferentes.

Depois, para respeitar a temporalidade dos registros, colocou-se a variável data de processamento em ordem ascendente

Tabela 1. Descrição de arquivos da Autorização de Procedimentos Ambulatoriais de Alta Complexidade

Tipo de arquivo Descrição Campos

Número da APAC e dados do paciente e do procedimento principal autorizados, como

$\mathrm{PF}^{*}$

$\mathrm{TA}^{*}$
Dados de Registro da APAC

Dados de faturamento do procedimento nome, nome da mãe, CPF, data de nascimento, sexo, local de residência, mês e ano de início do tratamento, diagnóstico principal e secundário (relacionados ao procedimento), número da APAC, data de início e fim da validade da APAC, data de processamento. Número da APAC, CPF do paciente e procedimento principal, datas de processamento e realização do procedimento, quantidade produzida (apresentada), quantidade aprovada e status da aprovação

*Estes nomes podem variar de acordo com a origem das bases (DATASUS, secretarias de saúde etc.) ou dos anos escolhidos para avaliação; APAC: autorização de procedimentos ambulatoriais de alta complexidade; PF: arquivo de pacientes que recebem medicamentos excepcionais; TA: arquivo de ocorrências de APAC 
com o comando sort do programa SPSS. Em seguida, utilizouse o comando restructure variables to cases usando as variáveis: número de APAC e procedimento principal para a identificação dos casos. Como resultado, cada linha representava uma APAC com seus procedimentos e até três respectivas datas de processamento, além dos outros campos utilizados nas análises. O mesmo processo foi realizado para a base TA.

Nos casos de APACs com mesmo número de registro e procedimentos diferentes, pode se realizar uma nova reestruturação dos dados usando o mesmo comando restructure variables to cases, mas somente com a variável com o número de APAC como variável identificadora Isso permitiria a identificação e agrupamento desses registros, caso existentes. A colocação das datas de processamento em ordem crescente para manter a ordem temporal dos registros teve de ser reproduzida em cada etapa usando o comando sort-ascendent order.

A junção das duas bases PF e TA através do comando merge files - add variables foi realizada usando como identificador único o número das APACs. Os registros de uma base que não possuíam correspondência na outra base foram excluídos da análise.

\section{5 atapa: Rotina de duplicidade para criar} um número identificação única do paciente

Os campos CPF do paciente e do Cartão Nacional de Saúde apresentavam um grande número de dados inconsistentes ou faltantes, o que impossibilitava a utilização desses campos em relacionamento determinístico. Portanto, pela ausência de um identificador único individual confiável nas bases da APAC, a criação da base centrada no indivíduo foi realizada através da rotina de duplicidade do software RecLink 3.

Essa rotina utiliza a técnica de relacionamento probabilístico para identificação de registros duplicados em uma mesma base de dados que não possui identificador único. $O$ processo é similar ao processo de relacionamento de registros com bases diferentes, ou seja, passa pelas etapas de padronização, blocagem, pareamento e revisão manual dos pares para classificação de pares verdadeiros ou não ${ }^{17,18}$.

Para a estratégia de blocagem, ou seja, criação de conjuntos comuns de registros, segundo uma ou mais chaves de identificação, e para otimização do processo de comparação, utilizamos três chaves formadas pela associação de campos a seguir: sexo do paciente+código soundex do primeiro nome do paciente+código soundex do último nome do paciente; sexo do paciente+código soundex do primeiro nome do paciente; sexo do paciente+código soundex do último nome do paciente.

Neste estudo, os principais campos utilizados na revisão manual para confirmação dos pares foram o nome do paciente, data de nascimento, primeiro nome da mãe e CPF. Outros campos utilizados na revisão manual para confirmação dos pares verdadeiros foram o nome completo da mãe, endereço e CEP.

Após a confirmação dos pares, o programa cria dois arquivos, um reduzido com uma entrada para cada indivíduo e outro mantendo os arquivos duplicados. Em ambos os arquivos o programa adiciona um campo com um número identificador único pra cada indivíduo. Pela necessidade de mantermos as múltiplas entradas do indivíduo para a avaliação de adesão, usamos o arquivo com os registros duplicados para a próxima etapa.

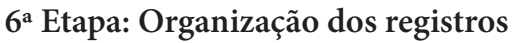
em uma base centrada no indivíduo

Nessa última etapa, para a organização das reentradas em ordem temporal e em uma linha única por paciente, as datas de início de cada APAC foram colocadas em ordem crescente e novamente se utilizou o comando restructure variables to cases do programa SPSS usando o número identificador criado no passo anterior.

Aqueles pacientes que não possuíam nenhum código de doença relacionado à doença de Alzheimer ou não apresentavam dados suficientes para a criação das variáveis necessárias para essa avaliação foram excluídos. Como para esse estudo o tempo de acompanhamento para avaliação de adesão foi determinado em no mínimo seis meses, os pacientes que entraram no programa nos últimos seis meses de 2005 também foram excluídos.

Durante o processo de relacionamento e construção da base, foi criada uma única variável identificadora do paciente, o que permitiu que todas as variáveis com informação sensível fossem retiradas antes do processo de análise. Este estudo foi autorizado pelo Comitê de Ética e Pesquisa do Instituto de Medicina Social da Universidade do Estado do Rio de Janeiro (UERJ).

\section{RESULTADOS}

Após realização das seis etapas de processamento, foi obtida a base cuja unidade de análise é o indivíduo, contendo os dados demográficos, unidade de dispensação, as múltiplas entradas de abastecimento com suas respectivas datas de apresentação, código primário e secundário do CID, o procedimento realizado (nesse caso medicamento e dose por unidade de apresentação) e número de unidades dispensadas. Para melhor compreensão, a Figura 2 apresenta os resultados após cada etapa de processamento e os registros excluídos no processo.

A base final possuía 756 pacientes com possibilidade de ao menos 6 meses de acompanhamento a partir do reabastecimento de suas receitas.

Através dessas múltiplas entradas, podem ser calculadas medidas de adesão já validadas na literatura mundial ${ }^{19-21}$. Alguns exemplos dessas medidas, sua descrição e formas de cálculos das mesmas são apresentados na Tabela 2. 


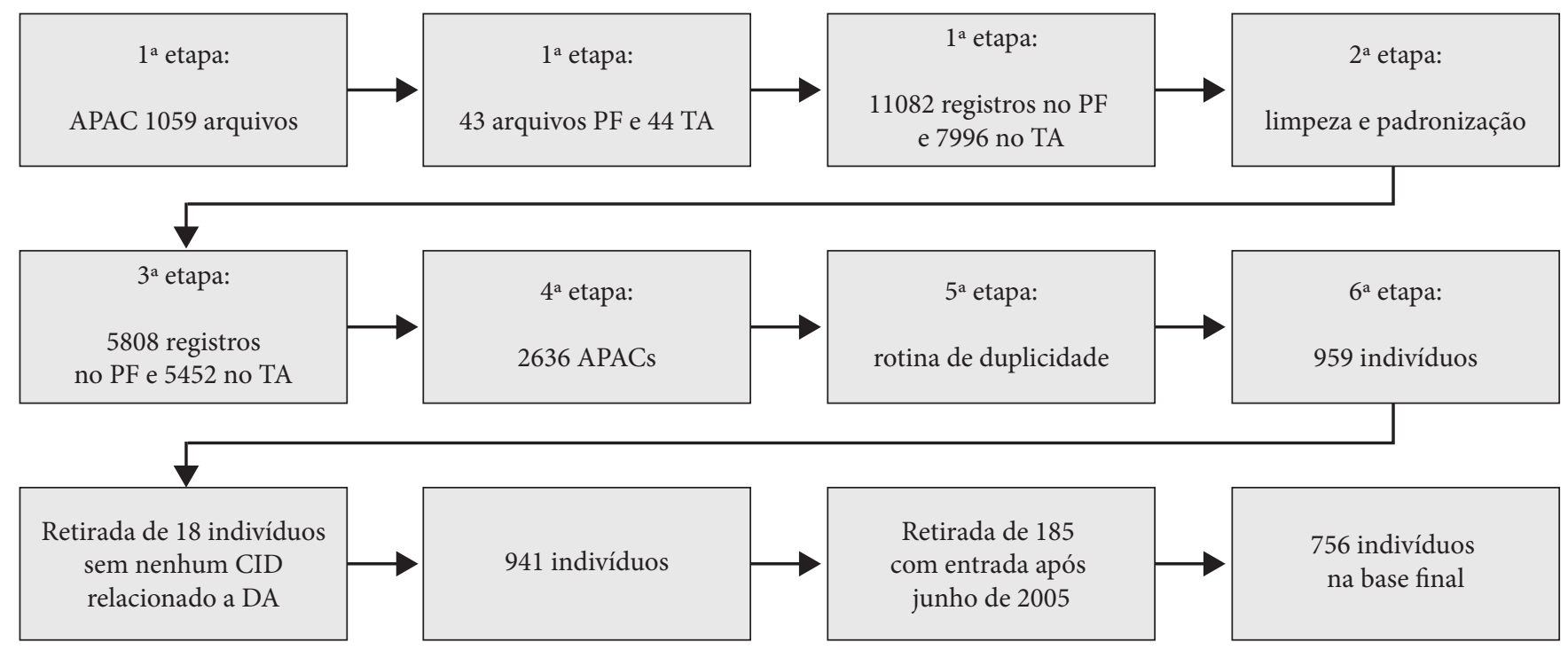

APAC: autorização de procedimentos ambulatoriais de alta complexidade; PF: arquivo de pacientes que recebem medicamentos excepcionais; TA: arquivo de ocorrências de APAC; CID: classificação estatística internacional de doenças e problemas relacionados com a saúde; DA: Doença de Alzheimer

Figura 2. Diagrama dos resultados de cada etapa de processamento e registros excluídos

Tabela 2. Descrição das medidas de adesão e exposição as medicações utilizadas no estudo

$\begin{array}{ll}\text { Tipo de medida } & \text { Descrição } \\ \begin{array}{l}\text { Número de } \\ \text { reabastecimentos }\end{array} & \begin{array}{l}\text { Soma do número de entradas únicas que o paciente tem } \\ \text { na base (ou número de datas únicas de apresentação) até } \\ \text { a data final do estudo }\end{array} \\ \begin{array}{l}\text { Tempo em posse do } \\ \text { medicamento ou dias } \\ \text { cobertos pela prescrição }\end{array} & \begin{array}{l}\text { Número de reabastecimentos do paciente é } \\ \text { multiplicado pelo tempo provável de tratamento } \\ \text { recebido em cada reabastecimento } \\ \text { Tempo de }\end{array} \\ \begin{array}{l}\text { Número de dias entre a data inicial da primeira APAC } \\ \text { (data índex) até a data de apresentação da última APAC } \\ \text { Persistência }\end{array} & \begin{array}{l}\text { mais o número aproximado de dias de tratamento dados } \\ \text { neste último reabastecimento ou até a data de óbito. }\end{array}\end{array}$

Proporção ou Taxa de Abandono

Proporção de posse de medicamento

Adesão ao
reabastecimento do
medicamento de
cada paciente

Dose total acumulada

Dose média diária
Proporção de pacientes que a data do último abastecimento ocorre antes de completar um período determinado (ex.: seis meses após a data índex).

Total de dias coberto pela prescrição dividido pelo número de dias de seguimento para cada indivíduo e multiplicado por 100. O PPM varia de zero a um e indica a proporção de tempo que o paciente está em posse do medicamento sobre a duração total de tratamento Total de dias coberto pela prescrição dividido pela duração total de acompanhamento e multiplicado por 100. Essa medida é expressa em percentual e indica a proporção de tempo que o paciente está em posse do medicamento sobre a duração total do estudo ou sobre tempo pré-determinado pelo investigador.

Número de unidades (comprimidos ou solução) versus a dosagem desta unidade

Dose total acumulada dividida por número de dias em posse do medicamento

\section{Comentários}

Número de vezes que o paciente procura o serviço de saúde para reabastecimento de sua receita

Número de dias que o paciente tem o medicamento acessível

Número de dias que o paciente continua em contato com o programa ou serviço de saúde. Não considera intervalos de acesso e uso que possam ter ocorrido no período

Pode ser calculada como uma proporção ou uma taxa (incidência mensal de abandono).

Após a data do último reabastecimento pode se acrescentar uma janela de tempo (30/60/90 dias) para que se considere o medicamento recebido no último reabastecimento e/ou medicamento armazenado.

Proporção do tempo de acompanhamento que o paciente tem o medicamento acessível.

Podem ser categorizada (ex.: $\geq 80 \%$ - aderente; $50-80 \%$ parcialmente aderente; $<50 \%$ não aderente)

Proporção de um pré-determinado período que o paciente tem o medicamento acessível.

Podem ser categorizada (ex.: $\geq 80 \%$ - aderente; $50-80 \%$ parcialmente aderente; $<50 \%$ não aderente)

Nível de exposição total do paciente ao medicamento

Nível de exposição média diária do paciente ao medicamento

PPM: Proporção de posse de medicamento; ARM: Adesão ao reabastecimento do medicamento de cada paciente; APAC: autorização de procedimentos ambulatoriais de alta complexidade 


\section{DISCUSSÃO}

A utilização da combinação dos métodos determinístico e probabilístico, de relacionamento nas bases de dados do componente APAC do sistema de informação ambulatorial permitiu a construção de uma base com dados de uma coorte longitudinal de pacientes em uso do programa de assistência farmacêutica em doença de Alzheimer do Sistema Único de Saúde. Esse tipo de coorte abre possibilidade de vários tipos de análises, entre eles o monitoramento da adesão dos indivíduos no programa através de várias medidas, já consagradas na literatura, que focam no reabastecimento de receitas.

O uso das informações do subsistema APAC sem aplicação desse tipo de metodologia pode ser importante na avaliação das tendências e características gerais do consumo de medicamentos e gastos em saúde, mas são limitados quando um conhecimento maior da população usuária é necessário.

Os resultados deste estudo apenas ratificam essa limitação, já que mostram uma grande diferença entre o número de registros e procedimentos e o número de pacientes que são atendidos. Com isso, só se corrobora a necessidade de novas metodologias ou inclusão no sistema de informação de variáveis que facilitem essa transformação de uma base centrada em registros para uma base centrada em indivíduos.

$\mathrm{O}$ uso a metodologia de relacionamento de bases de dados na construção de uma coorte de pacientes ${ }^{18,22,23}$, apesar de já ter sido abordada na literatura nacional, teve, neste estudo, foco orientado para a observação das múltiplas entradas do paciente na base para dispensação de medicamentos e intervalos entre essas entradas. Isso traz uma maior necessidade de atenção à ordenação e temporalidade dessas reentradas e inclusão dos arquivos com dados dos medicamentos e doses.

Além dos sugeridos, essa metodologia também permite incluir informações sobre: os intervalos entre os reabastecimentos, as quantidades dispensadas e registros com quantidades zeradas. Essa inclusão permitiria outras avaliações como identificação de pacientes de maior irregularidade de acesso ao sistema, avaliação da real dose de medicamento dispensada ao indivíduo, como também períodos em que esse medicamento não foi efetivamente dispensado.

Essa construção não é um processo simples e, durante o processo de transformação dos arquivos, nos deparamos com algumas dificuldades como: grande número de registros duplicados, dados inconsistentes e múltiplos procedimentos em uma mesma APAC. Essas dificuldades só reforçaram a necessidade de aprimorar o conhecimento não só da base como o processo que a alimenta. Outras limitações encontradas foram a falta parcial de correspondência entre os registros do arquivo PF e TA e o grande número de registros do arquivo TA com campos de unidades solicitadas ou fornecidas não preenchidos ou com o valor zero.
Neste estudo, optou-se pela exclusão de todos os registros que não estivessem em ambas as bases e de todos os registros com zero no número de unidades fornecidas. Essa opção, apesar de garantir que só sejam analisados os reabastecimentos que realmente ocorreram, poderá acarretar em um viés de informação, pois estes registros podem estar relacionados a um problema de desabastecimento ou abastecimento irregular das farmácias que atendem a esses indivíduos. Esse viés poderia superestimar a qualidade do serviço oferecido pelo sistema de saúde, como também aumentar o papel do indivíduo como um dos principais atores na falta de adesão. Uma cuidadosa reflexão dessas limitações deve ocorrer antes da escolha das medidas de adesão e uso de medicamentos a serem utilizados.

Mais ainda, como essa base não foi desenvolvida com finalidade de pesquisa, a acurácia dos dados é geralmente considerada menor quando comparada a estudos com coleta primária de dados. Além disso, a literatura mostra resultados conflitantes na avaliação de concordância entre as medidas baseadas em reabastecimento e aquelas baseadas em autorrelato ${ }^{24-26}$ ou monitoramento eletrônico ${ }^{27}$.

Apesar disso, a associação dessas medidas com desfechos econômicos e clínicos (incluindo mortalidade) tem sido consistente $^{2,28-30}$. Ademais, tais bases permitem a análise de grande número de indivíduos, o que seria difícil de ser conseguido com o seguimento ativo de uma coorte, gerando informação para apoiar a gestão do setor saúde.

O uso de dados secundários em saúde tem aumentado consideravelmente nos últimos anos como consequência do aumento da disponibilização de bases de dados e da possibilidade de cobrir grandes populações. Com isso, novos métodos têm surgido para lidar com as limitações inerentes ao uso desses dados, permitindo a avaliação de um grande número de dados em curto espaço de tempo e o aumento no número de programas de informática que facilitam a análise e o relacionamento entre diferentes sistemas de informação. A análise das informações disponibilizadas por essas bases públicas tem se tornado uma importante ferramenta para a vigilância e monitoramento de ações e políticas públicas ${ }^{31-33}$.

Embora pouco utilizada, a avaliação sistemática da adesão aos programas de assistência abre várias possibilidades tanto no campo da epidemiologia como no de planejamento em saúde. A partir da análise da adesão, torna-se possível a identificação precoce de uma população com maior risco, a avaliação indireta da qualidade de um programa, a avaliação da logística e do armazenamento de informações, entre outras aplicações. Com o crescente interesse na análise de bases de dados secundários, esperamos que tanto as metodologias de análise de adesão como a qualidade dessas bases sejam aprimoradas num futuro próximo. 


\section{REFERÊNCIAS}

1. World Health Organization (WHO). Adherence to long-term therapies: evidence for action. Geneva: World Health Organization; 2003.

2. Reginster JY. Adherence and persistence: impact on outcomes and health care resources. Bone. 2006;38(2 Suppl 2):S18-21.

3. Oleen-Burkey MA, Dor A, Castelli-Haley J, Lage MJ. The relationship between alternative medication possession ratio thresholds and outcomes: evidence from the use of glatiramer acetate. J Med Econ. 2011;14(6):739-47.

4. Malta DC, Merhy EE. O percurso da linha do cuidado sob a perspectiva das doenças crônicas não transmissíveis. Interface Comunic Saude Educ. 2010;14(34):593-606.

5. Carias CM, Vieira FS, Giordano CV, Zucchi P. Medicamentos de dispensação excepcional: histórico e gastos do Ministério da Saúde do Brasil. Rev Saúde Pública. 2011;45(2):233-40.

6. Brandão CM, Guerra AA Jr, Cherchiglia ML, Andrade EI, Almeida AM, da Silva GD, de Queiroz OV, Faleiros DR, Acurcio Fde A. Gastos do Ministério da Saúde do Brasil com medicamentos de alto custo: uma análise centrada no paciente. Value Health. 2011;14(5 Suppl):S71-7.

7. Lima-Dellamora EC, Caetano R, Osorio-de-Castro CGS. Dispensação de medicamentos do componente especializado em polos no Estado do Rio de Janeiro. Ciênc Saúde Col. 2012;17(9):2387-96.

8. Brasil. Ministério da Saúde. Secretaria de Vigilância em Saúde. Departamento de DST Aids e hepatites virais. Adesão ao tratamento antirretroviral no Brasil: coletânea de estudos do projeto ATAR. Brasília: Ministério da Saúde; 2010.

9. Brasil. Ministério da Saúde. Secretaria de Vigilância em Saúde. Departamento de DST Aids e hepatites virais. Protocolo de assistência farmacêutica em DST/HIV/Aids: Recomendações do Grupo de Trabalho de Assistência Farmacêutica. Brasília: Ministério da Saúde; 2010.

10. Fairman K, Motheral B. Evaluating medication adherence: Which measure is right for your program? J Managed Care Pharm. 2000;6(6):499-504

11. Bosworth HB. Medication adherence. In: Bosworth $\mathrm{H}$ (ed.). Improving patient treatment adherence - A clinician's guide. New York: Springer; 2010. p. 68-94.

12. Bosworth HB, Granger BB, Mendys P, Brindis R, Burkholder R, Czajkowski SM, Daniel JG, Ekman I, Ho M, Johnson M, Kimmel SE, Liu LZ, Musaus J, Shrank WH, Whalley Buono E, Weiss K, Granger CB. Medication adherence: a call for action. Am Heart J. 2011;162(3):412-24.

13. Krueger KP, Berger BA, Felkey B. Medication adherence and persistence: a comprehensive review. Adv Ther. 2005;22(4):313-56.

14. Gomes RRFM, Machado CJ, Acúrcio FA, Guimarães MDC. Utilização dos registros de dispensação da farmácia como indicador da não-adesão à terapia anti-retroviral em indivíduos infectados pelo HIV. Cad Saúde Pública. 2009;25(3):495-506.

15. Brasil. Ministério da Saúde. Secretaria de Atenção à Saúde. Portaria SAS nº. 768, de 26 de outubro de 2006. Brasília; 2006.

16. Brasil. Ministério da Saúde. Departamento de Informática do SUS. DATASUS. [Software]. Tab para Windows - TabWin. [cited 2013 Jun 20]. Available from: http://www2.datasus.gov.br/DATASUS/index.php?area=040805\&item =1

17. Camargo Junior KR, Coeli CM. Reclink: aplicativo para o relacionamento de bases de dados, implementando o método probabilistic record linkage. Cad Saúde Pública. 2000;16(2):439-47.

18. Queiroz OV, Guerra Junior AA, Machado CJ, Andrade ELG, Meira Junior W, Acúrcio FA, Santos Filho W, Cherchiglia ML. A construção da Base Nacional de Dados em Terapia Renal Substitutiva (TRS) centrada no indivíduo: relacionamento dos registros de óbitos pelo subsistema de Autorização de Procedimentos de Alta Complexidade (APAC/SIA/SUS) e pelo Sistema de Informações sobre Mortalidade (SIM) - Brasil, 2000-2004. Epidemiol Serv Saúde. 2009;18(2):107-120.

19. Hudson M, Rahme E, Richard H, Pilote L. Comparison of measures of medication persistency using a prescription drug database. Am Heart J. 2007;153(1):59-65.

20. Peterson AM, Nau DP, Cramer JA, Benner J, Gwadry-Sridhar F, Nichol M. A checklist for medication compliance and persistence studies using retrospective databases. Value Health. 2007;10(1):3-12.

21. Cramer JA, Roy A, Burrell A, Fairchild CJ, Fuldeore MJ, Ollendorf DA, Wong PK. Medication compliance and persistence: terminology and definitions. Value Health. 2008;11(1):44-7.

22. Teixeira CLS, Bloch KV, Klein CH, Coeli CM. Método de relacionamento de bancos de dados do Sistema de Informações sobre Mortalidade (SIM) e das autorizções de internação hospitalar (BDAIH) no Sistema Único de Saúde (SUS), na investigação de óbitos de causa mal-definida no Estado do Rio de Janeiro, Brasil, 1998. Epidemiol Serv Saúde. 2006;15(1):47-57.

23. Gomes Jr SCS, De Martino R, Almeida RT. Rotinas de integração das tabelas do sistema de autorizção de procedimentos de alta complexidade em oncologia do Sistema Único de Saúde. Cad Saúde Colet. 2003;11(2):231-54.

24. Guénette L, Moisan J, Préville M, Boyer R. Measures of adherence based on self-report exhibited poor agreement with those based on pharmacy records. J Clin Epidemiol. 2005;58(9):924-33.

25. Garber MC, Nau DP, Erickson SR, Aikens JE, Lawrence JB. The concordance of self-report with other measures of medication adherence: a summary of the literature. Med Care. 2004;42(7):649-52.

26. Cook CL, Wade WE, Martin BC, Perri M 3rd. Concordance among three self-reported measures of medication adherence and pharmacy refill records. J Am Pharm Assoc. 2005;45(2):151-9.

27. Hansen RA, Kim MM, Song L, Tu W, Wu J, Murray MD. Comparison of methods to assess medication adherence and classify nonadherence. Ann Pharmacother. 2009;43(3):413-22.

28. DiMatteo MR, Giordani PJ, Lepper HS, Croghan TW. Patient adherence and medical treatment outcomes: a meta-analysis. Med Care. 2002;40(9):794-811.

29. Parker JP, McCombs JS, Graddy EA. Can pharmacy data improve prediction of hospital outcomes? Comparisons with a diagnosis-based comorbidity measure. Med Care. 2003;41(3):407-19.

30. Balkrishnan R. The importance of medication adherence in improving chronic-disease related outcomes: what we know and what we need to further know. Med Care. 2005;43(6):517-20.

31. Malta DC, Cezário AC, Moura L, Morais Neto OL, Silva Junior JB. A construção da vigilância e prevencão das doenças crônicas não transmissíveis no contexto do Sistema Único de Saúde. Epidemiol Serv Saúde. 2006;15(3):47-65.

32. Rodrigues-Júnior AL. A inteligência epidemiológica como modelo de organização em saúde. Ciênc Saúde Coletiva. 2012;17(3):797-805.

33. Malta DC, Morais Neto OL, Silva Junior JB. Apresentação do plano de ações estratégicas para o enfrentamento das doenças crônicas não transmissíveis no Brasil, 2011 a 2022. Epidemiol Serv Saúde. 2011;20(4):425-38. 